Revista de Psicología de la PUCP. Vol. XXI, 2, 2003

\title{
Factores del hogar en el rendimiento escolar ${ }^{1}$
}

\author{
Yetilú de Baessa ${ }^{2}$ \\ Universidad del Valle de Guatemala
}

\author{
Francisco Javier Fernández ${ }^{3}$ \\ Universidad Central de Venezuela
}

\begin{abstract}
La presente investigación tuvo como objetivo examinar la relación que existe entre el rendimiento escolar y los factores asociados al hogar de alumnos de tercer grado de primaria que asisten a escuelas primarias localizadas en áreas urbanas y rurales de Guatemala. Se tomó al azar una muestra de 4,952 estudiantes ( $52 \%$ niños y $48 \%$ niñas) de tercer grado a nivel nacional. Se aplicaron pruebas de lectura y matemática y se obtuvo información de los factores asociados al rendimiento. Se analizaron los datos mediante dos técnicas estadísticas: análisis de regresión múltiple y análisis de correspondencia múltiple. Los resultados obtenidos muestran que un porcentaje importante de la varianza en el rendimiento, especialmente en lectura, se explica por ciertas variables relacionadas con el entorno del hogar. El resto, probablemente, se podría explicar por factores relacionados con el docente y con la escuela en sí, o por características individuales de los alumnos, que no pudieron ser medidos.

Palabras clave: rendimiento escolar, factores asociados al hogar.
\end{abstract}

\section{Home factors in academic achievement}

The objective of this research was to examine the relationship between academic achievement and home factors in third grade students attending urban and rural schools in Guatemala. The sample consisted of 4,952 students selected at random (52\% males and $48 \%$ females). Mathematics and reading tests were administered to the students and other associated factors were obtained through a questionnaire. The data was analyzed using two statistical approaches: multiple regression and analysis of multiple correspondence. Results showed that an important percentage of the variance in reading achievement is explained by the variables related to home environment. The rest is probably related to factors associated to the teacher or school per se or by individual characteristics of the students that were not possible to measure.

Key words: scholarship perfomance, home associated factors.

Trabajo basado en una ponencia presentada en el Congreso Latinoamericano de Psicología, Chile, 2001

2 Doctora en Psicología por la Universidad de Austin, USA. Decana de la Facultad de Psicología de la Universidad Francisco Marroquín, Guatemala. En la actualidad realiza investigaciones sobre "Identidad étnica entre estudiantes adolescentes". Dirección postal: 3 Calle 7-79, Zona 15, Ciudad de Guatemala, Guatemala. Correo electrónico: yetilu@ufm.edu.gt.

3 Doctor en Ciencias Sociales por la Universidad Francisco Marroquín, Guatemala. Docente/ Investigador de la Facultad de Economía y Ciencias Sociales de la Universidad Central de Venezuela. Realiza investigaciones dentro del programa "Crecimiento y desarrollo del niño 

En los estudios que se han llevado a cabo en Guatemala sobre rendimiento escolar ${ }^{4}$ (Baessa et al., 1999) se han analizado diferentes variables que inciden en el rendimiento escolar, en su mayoría relacionadas con la escuela, el docente y con el alumno. Sin embargo, durante el año 2000 se hizo un intento por analizar variables relacionadas con el entorno en el cual vive el alumno. Esto representaba un reto, dado el alto nivel de analfabetos que existen en el país (según datos reportados por el gobierno actual, alcanza un $40 \%$ ).

En la mayoría de estudios que se hacen sobre rendimiento escolar, se utiliza un cuestionario que se envía con los alumnos a los padres para explorar variables relativas al hogar. En Guatemala este procedimiento es casi imposible de hacer en las escuelas rurales, ya que el índice de analfabetismo alcanza a veces más de un $70 \%$, sobre todo en las madres. Debido a esto, se entrevistó a los alumnos en forma individual para lograr obtener información sobre el hogar.

El estudio incluyó este tema desde dos puntos de vista, uno tradicional, tratando de predecir lectura y matemática mediante análisis de regresión, y otro utilizando análisis de correspondencia múltiple.

A continuación se presenta la base teórica que fundamenta el estudio, la metodología, los resultados y su discusión.

y adolescente guatemalteco" y en "Identidad étnica entre estudiantes adolescentes". Dirección postal: lote 9, Manzana 4, Res. Villas de Montesano, Montensano, Zona 16, Ciudad de Guatemala, Guatemala. Correo electrónico: arauquin@ufm.edu.gt.

La evaluación a nivel nacional ha sido financiada por el Ministerio de Educación de Guatemala 
Yetilú de Baessa y Francisco Javier Fernández

\section{Marco Teórico}

La generalidad de estudios que se han hecho en América Latina (Laboratorio Latinoamericano de Evaluación de la Calidad de la Educación, 2000) sobre rendimiento escolar y factores asociados, ha enfocado aspectos relacionados con el hogar. Una de las variables que tiene mayor valor predictivo es la educación de los padres y, en los estudios realizados en Guatemala, la educación de la madre parece tener mayor importancia que la educación del padre (Baessa et al., 1999).

Sin embargo, en otras variables tales como si los alumnos ven televisión y cuántas horas de exposición tienen, se han encontrado resultados contradictorios (Clarke \& Kurtz-Costes, 1997; Cooper, Valentine, Nye \& Lidsay, 1999). En algunos artículos, los autores mencionan que la televisión tiene una relación inversa con el rendimiento académico y en otros informan lo opuesto, es decir, que la televisión ayuda a los alumnos a mejorar su rendimiento. Otras de las variables asociadas que a menudo se han investigado es la presencia y utilización de material de lectura en el hogar (Halle, Kurtz-Costes \& Mahoney, 1997).

\section{Objetivos}

El objetivo general es el siguiente:

- Examinar en qué medida las variables que se relacionan con el hogar afectan el rendimiento de los alumnos de tercer grado de las escuelas y colegios en una muestra tomada al azar en toda Guatemala.

Los objetivos específicos son los siguientes:

- Establecer cuáles son las variables del hogar que inciden en el rendimiento del niño en las escuelas del área urbana y rural. 
- Establecer cuáles son las variables del hogar que inciden en el rendimiento del niño en las escuelas públicas y colegios privados.

\section{Metodología}

\section{Participantes}

Se seleccionó una muestra al azar de 4952 estudiantes (51.9\% niños y $48.1 \%$ niñas) de tercer grado a nivel nacional, los cuales habían sido evaluados en la evaluación que hizo el Ministerio de Educación de Guatemala en lectura y matemática en tercero y sexto grado de primaria en el año 2001.

\section{Instrumentos}

A fin de obtener los datos requeridos para la investigación, se elaboró una hoja de información del alumno, la cual fue aplicada por el personal de investigación a cinco niños seleccionados al azar en cada uno de los establecimientos en estudio.

La hoja de información del alumno está dividida en tres partes: la primera de ellas es sobre el alumno propiamente (identidad étnica, si habla, lee y escribe en lengua materna, si asistió a preescolar, si ha repetido grados, si recibe ayuda de algún familiar para hacer los deberes escolares); la segunda se relaciona con el nivel educativo de los padres, y la tercera se relaciona con los hábitos de lectura y entretenimiento en el hogar (si leen libros o periódicos, quiénes los leen; si ven televisión, quiénes la ven y cuántas horas).

\section{Procedimiento}

Con el objeto de tener una mayor perspectiva de los factores que inciden en el rendimiento del escolar en sus estudios, se decidió aplicar dos formas de análisis basadas en lo siguiente: 
- La correlación y la regresión múltiple mediante el paquete estadístico SPSS.

- El análisis de correspondencia múltiple por medio del paquete SPAD.N.

\section{Resultados}

\section{Análisis de Regresión Múltiple}

En la Figura 1 se muestran los resultados de los alumnos que asisten a escuelas urbanas comparándolos con los que asisten a escuelas rurales.

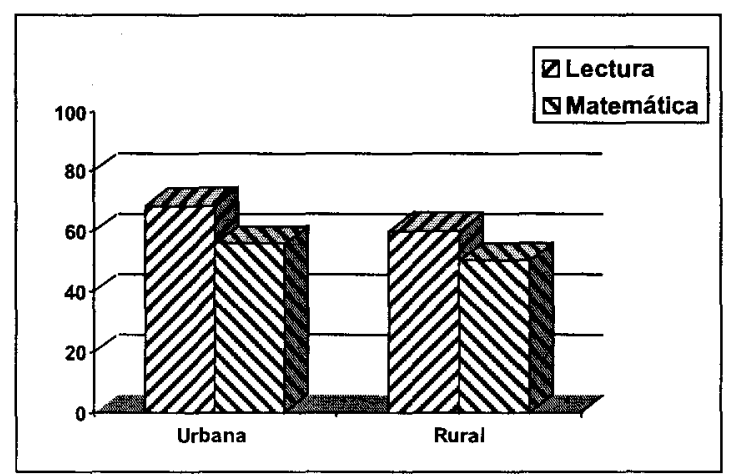

Figura 1. Comparación entre escuelas urbanas y rurales en tercer grado.

Tanto en lectura como en matemática las diferencias de medias son estadísticamente significativas. Por ese motivo, se analizaron los datos de manera separada en los análisis de regresión.

Los primeros análisis que se hicieron en este estudio en particular fueron obtener las correlaciones entre el rendimiento en lectura y matemática de los alumnos de tercer grado y las variables asociadas al hogar que se habían medido durante la evaluación a nivel nacional. 
Se utilizó un modelo de regresión por pasos, en el cual entran en la regresión las variables de acuerdo con la correlación que tienen con la variable predicha. A continuación se presentan los coeficientes de determinación que se encontraron en cada modelo calculado, en función de cada variable que entraba en el modelo.

En el caso de la predicción de lectura en escuelas urbanas, las variables que entraron en la recta de regresión fueron: si el alumno habla o no un idioma maya; el grado de educación de la madre; la cantidad de libros que tienen en el hogar; si ven o no ven televisión, y si los padres leen libros al niño (Cuadro 1).

\section{Cuadro 1}

Resumen del modelo para predecir lectura en escuelas urbanas

\begin{tabular}{|lllc|}
\hline Modelo & $R$ & $R^{\prime \prime}$ & $\begin{array}{c}\text { Cambio } \\
\text { en } R^{\prime \prime}\end{array}$ \\
\hline (constante) Habla maya & .162 & .161 & .161 \\
(constante) Escolaridad de la madre & .212 & .212 & .051 \\
(constante) Cantidad libros & .238 & .237 & .025 \\
(constante) TV & .251 & .250 & .013 \\
(constante) Padres leen libros & .257 & .256 & .006 \\
\hline
\end{tabular}

El modelo explica aproximadamente un $26 \%$ de la varianza en el rendimiento en lectura y no hay ningún cambio importante al agregar la variable relativa a si los padres leen libros a sus hijos.

El Cuadro 2 muestra el modelo que se usó para predecir rendimiento matemático en alumnos de escuelas urbanas. 


\section{Cuadro 2}

Resumen del modelo para predecir matemática en escuelas urbanas.

\begin{tabular}{|lccc|}
\hline Modelo & $R$ & $R^{\prime \prime}$ & $\begin{array}{c}\text { Cambio } \\
\text { en } R^{\prime \prime}\end{array}$ \\
\hline (constante) Escolaridad de la madre & .226 & .051 & .051 \\
(constante) Habla maya & .271 & .073 & .022 \\
(constante) Cantidad libros & .297 & .088 & .015 \\
(constante) Horas TV & .303 & .092 & .004 \\
(constante) Padres leen libros & .308 & .095 & .003 \\
\hline
\end{tabular}

En el caso de matemática, el mejor modelo de regresión solamente pudo predecir el $9.3 \%$ de la varianza. Es decir, en la predicción del aprendizaje de la matemática intervienen muchas otras variables que probablemente no se relacionan con el hogar.

\section{Cuadro 3}

Resumen del modelo para predecir lectura en escuelas rurales.

\begin{tabular}{|lccc|}
\hline Modelo & $R$ & $R^{\prime \prime}$ & $\begin{array}{c}\text { Cambio } \\
\text { en } R^{\prime \prime}\end{array}$ \\
\hline (constante) Habla maya & .451 & .204 & .204 \\
(constante) TV & .495 & .245 & .041 \\
(constante) Escolaridad de la madre & .506 & .256 & .011 \\
(constante) Leen periódico & .510 & .260 & .004 \\
\hline
\end{tabular}

Al analizar el resumen del modelo para predecir lectura en las escuelas rurales, se observa que sólo cuatro variables asociadas con el hogar predicen $26 \%$ de la varianza en el rendimiento en lectura (Cuadro 3). El orden en el cual entran los predictores al modelo no es igual que en el caso de los alumnos que asisten a las escuelas urbanas. Se observa que la escolaridad de la madre no tiene tanto peso, probablemente por la poca variabilidad que tiene esa variable en el sector rural.

A continuación, en el Cuadro 4, aparece el modelo de regresión múltiple que se usó para predecir el rendimiento en matemática de los alumnos de tercer grado que asisten a las escuelas rurales. 
Factores del hogar en el rendimiento escolar

\section{Cuadro 4}

Resumen del modelo para predecir matemática en escuelas rurales.

\begin{tabular}{|lccc|}
\hline Modelo & $\mathrm{R}$ & $\mathrm{R}^{\sim}$ & $\begin{array}{c}\text { Cambio } \\
\text { en } \mathrm{R}^{\prime \prime}\end{array}$ \\
\hline (constante) Habla maya & .217 & .047 & .047 \\
(constante) TV & .263 & .069 & .022 \\
(constante) Leen periódico & .273 & .075 & .006 \\
(constante) Escolaridad de la madre & .279 & .078 & .003 \\
\hline
\end{tabular}

Al igual que en el caso del modelo de regresión para predecir el rendimiento en matemática en las escuelas urbanas, la varianza explicada en el caso de los alumnos de escuelas rurales es baja (sólo 7.8\%) en relación con lectura.

\section{Análisis de Correspondencia Múltiple}

El Análisis de Correspondencia Múltiple es una técnica que responde a la necesidad de profundizar en las relaciones de dependencia que se establecen entre las variables cualitativas (nominales y ordinales) observadas en una misma población y, en particular, insistiendo en explicar cómo los distintos valores o categorías de las variables se relacionan entre sí (Cornejo, 1988) reduciéndolas en un conjunto de factores cuantitativos a través del cálculo de la distancia de Benzecri, el cual está basado en la Chi cuadrada. El análisis se llevó a cabo con el paquete estadístico francés denominado SPAD.N, versión 3.0 (Sistema Portable para el Análisis de Datos) (CISIA, 1993).

En este tipo de análisis se correlacionan todas las variables del estudio a través del análisis de correspondencias múltiples. El análisis se realiza siguiendo las recomendaciones de Crivisqui (1993), quien plantea que la estrategia a seguir en la interpretación de los resultados del Análisis Factorial de Correspondencias Múltiples debería contemplar los siguientes puntos:

- Estudio de la inercia asociada a los factores.

- Interpretación de los ejes.

Grado de generalidad de los factores.

Estudio de las variables. 
Coordenadas y contribuciones de los elementos activos.

- Interpretación de los Planos Factoriales (visualización de la agrupación de factores).

\section{Análisis de la Inercia}

En el presente estudio se manejaron 18 variables activas y una variable ilustrativa, las cuales en conjunto conformaron inicialmente un total de 71 modalidades. Sobre estas modalidades se aplicó un filtro con el fin de eliminar aquellas que presentaban muy bajo peso (menos del $2 \%$ ) y que podrían alterar o deformar la inercia global. Una vez culminado el procedimiento, el análisis de la inercia quedó constituido sobre la base de 64 modalidades. Las variables que se utilizaron en el estudio fueron las siguientes:
Variables Activas
1. Edad
2. Sexo
3. Calificación en lectura
4. Calificación en matemática
5. Estudios de pre-primaria
6. Con quién vive el niño
7. Quién ayuda al niño a hacer los deberes en el hogar
8. Escolaridad de la madre
9. Escolaridad del padre
10. Los padres leen libros en el hogar
11. Los padres leen el diario en el hogar
12. Leen el diario todos los días
13. Cuántas horas al día ven televisión
14. Tienen libros en casa
15. Cantidad de libros en la casa
16. Ubicación del centro educativo
17. Tipo de establecimiento
Variable Ilustrativa
1. Departamento 
La inercia global ${ }^{5}$ (Cuadro 5) de la masa de variables activas es de 2.556 , descompuesta sobre un total de 46 factores principales de alargamiento de las nubes de puntos-modalidades e individuos. De estos 46 ejes, los primeros 20 presentan valores propios (o autovalores superiores al valor promedio $2.556 / 46=0.0556$ ). En este estudio, el análisis se realizó sobre los dos primeros ejes factoriales, que aportan la información más relevante.

Estos ejes presentan una tasa de inercia o variabilidad acumulada de $14.93 \%$ (9.58\% y $5.35 \%$, respectivamente), con valores propios (o autovalores) de 0.2449 para el primer eje y de 0.1367 para el segundo eje.

\section{Cuadro 5}

Histograma de los nueve primeros valores propios.

\begin{tabular}{|ccccl|}
\hline Eje & $\begin{array}{c}\text { Valor } \\
\text { propio }\end{array}$ & $\%$ & $\begin{array}{c}\% \\
\text { Acumulado }\end{array}$ & \multicolumn{1}{c|}{ Histograma } \\
\hline 1 & .2449 & 9.58 & 9.58 & $* * * * * * * * * * * * * * * * * * * * * * * *$ \\
2 & .1367 & 5.35 & 14.93 & $* * * * * * * * * * * * * * * * * * * * *$ \\
3 & .0932 & 3.65 & 18.58 & $* * * * * * * * * * * * * * * * * * *$ \\
4 & .0888 & 3.48 & 22.06 & $* * * * * * * * * * * * * * * *$ \\
5 & .0824 & 3.22 & 25.26 & $* * * * * * * * * * * * * *$ \\
6 & .0775 & 3.03 & 28.32 & $* * * * * * * * * * *$ \\
7 & .0759 & 2.97 & 31.38 & $* * * * * * * * * *$ \\
8 & .0703 & 2.75 & 34.03 & $* * * * * * * *$ \\
9 & .0675 & 2.64 & 36.68 & $* * * * *$ \\
\hline
\end{tabular}

\section{Análisis de los Ejes}

En la formación del primer eje factorial (con el 9.58\% de la inercia o variabilidad total) las variables que más contribuyen son:

1. cantidad de libros que poseen en la casa (10.8\%),

2. grado de instrucción de la madre $(9.8 \%)$,

3. escolaridad del padre (8.0\%),

4. los padres leen el periódico diariamente $(7.9 \%)$, 
5. los padres leen periódico $(7.8 \%)$,

6. poseen libros en casa $(7.3 \%), \mathrm{y}$

7. área del estudio (6.6\%).

En relación con las modalidades, el primer eje factorial se construye en función de dos grupos: el primero de ellos, ubicado en el semi-eje negativo, indica que las edades de los niños fluctúan entre los 6 y 9 años, han estudiado pre-primaria, los padres tienen estudios a nivel diversificado, las madres tienen estudios de nivel básico y diversificado. Estas modalidades corresponden al área urbana, principalmente al área metropolitana, y en la actualidad estos niños estudian en centros educativos privados, reciben ayuda de sus madres en la realización de las tareas escolares en casa, los resultados que obtuvieron en la prueba de rendimiento en lectura estuvieron entre los 80 y 100 puntos, en el hogar tienen libros (más de 11), los padres leen libros a los niños, y leen el periódico en casa diariamente.

Por otro lado, en el semi-eje positivo se agrupan las modalidades que describen a niños entre 12 y 13 años de edad, cuyas puntuaciones en la prueba de lectura estuvieron entre los 20 y 59 puntos. En la prueba de matemática, los punteos están por debajo de los 40 puntos, los niños no han asistido a la escuela pre-primaria y estudian en centros educativos oficiales. El padre no estudió y se desconoce si la madre lo ha hecho. No poseen libros en casa, no leen periódicos, no ven televisión ni son ayudados en casa a hacer la tarea escolar.

En la formación del segundo eje factorial (con el 5.35\% de la inercia o variabilidad total) las variables que más contribuyen a su formación son:

1. el padre lee libros al niño diariamente (13.7\%),

2. el padre lee libros (13.4\%),

3. cantidad de libros que poseen en la casa (11.0\%),

4. nivel de instrucción del padre (10.3\%), 
5. nivel de instrucción de la madre $(9.2 \%)$,

6. los padres leen el periódico diariamente (7.9\%),

7. los padres leen periódico (7.8\%), y

8. poseen libros en casa $(7.3 \%)$.

Las modalidades que contribuyen con mayor significado a este eje marcan una polarización en relación con el nivel educativo de los padres, el rendimiento en lectura y matemática, la lectura de libros y periódicos en casa, el área de estudio de los padres, y el tipo de establecimiento educativo. Encontramos en el semi-eje positivo a aquellos jóvenes entre 10 y 11 años, cuyos padres poseen un nivel educativo entre primero y sexto grado, y sus madres, entre primero y tercer grado de primaria. Estos jóvenes pertenecen al área rural del sector educativo oficial y su rendimiento se encuentra entre 10 y 49 puntos en matemática y 40 y 59 puntos en lectura. Dentro de este mismo eje se observa que en la casa de este tipo de alumno se lee periódicos y libros. Por otro lado, el semi-eje negativo muestra a niños entre los 6 y 9 años cuyos padres poseen un nivel de instrucción diversificado. Su rendimiento es superior a los 60 puntos en matemática y a los 80 puntos en lectura, asisten al sector educativo privado y, son principalmente del área urbana. Al mismo tiempo se encuentra un grupo de niños que desconoce el nivel educativo del padre y no poseen libros en la casa.

\section{Interpretación de los Planos Factoriales I-II}

El análisis del Plano Factorial I-II refleja la presencia de dos grupos caracterizados por lo siguiente:

Grupo 1. Son jóvenes entre 10 y 13 años cuyo rendimiento en matemática estuvo por debajo de 40 puntos y en lectura entre 20 y 40 puntos. Asisten a escuelas del sector oficial y son predominantemente del área rural, no tienen estudios de pre-primaria y no son ayudados a hacer los deberes escolares en el hogar. En relación a los padres, éstos 
tienen un nivel educativo inferior o igual al tercer grado de primaria, en el hogar no leen el periódico, no tienen libros y no ven televisión.

Grupo 2. Está formado por niños entre 6 y 9 años, cuyos resultados en matemática estuvieron por encima de los 60 puntos y en lectura por encima de los 80 puntos. Asisten a establecimientos educativos del sector privado del área urbana y del área metropolitana, han realizado estudios de pre-primaria y son ayudados por ambos padres en la realización de las tareas escolares en el hogar. En cuanto al nivel de instrucción de los padres, el padre posee un nivel de instrucción entre cuarto y sexto de primaria y/o diversificado; la madre posee un nivel de instrucción básico y/o diversificado. En el hogar se posee libros, los padres leen periódicos y leen libros a los niños.

\section{Discusión}

\section{Resultados de los análisis de regresión múltiple}

El objetivo central de este estudio era establecer la relación que existe entre el rendimiento escolar y los factores asociados al hogar de alumnos de tercer grado de primaria que asisten a las escuelas primarias localizadas en áreas urbanas y rurales, en Guatemala. Se aplicaron pruebas de lectura y de matemática y se obtuvo información de factores asociados. Con el propósito de dar respuesta a esta pregunta central, se analizaron los datos mediante dos técnicas estadísticas: análisis de regresión múltiple y análisis de correspondencia múltiple.

Los análisis de regresión múltiple se hicieron separadamente para las escuelas del área urbana y para las del área rural. Se encontró que, en general, tanto en escuelas urbanas como en rurales los factores relacionados con el hogar predicen aproximadamente un $25 \%$ de la varianza del rendimiento en lectura. En términos generales dicho porcentaje es moderadamente alto, tomando en cuenta la gran 
cantidad de factores que pueden afectar el rendimiento académico de un estudiante. Sin embargo, la predicción del rendimiento en matemática es mucho más baja, no alcanza un $10 \%$ de la varianza.

Es interesante notar que las variables que entran en el modelo de regresión del rendimiento de alumnos que asisten a escuelas rurales no son iguales al modelo que predice el rendimiento en el área urbana para lectura y matemática. Las condiciones sociales y económicas prevalecientes en el área urbana son completamente diferentes a las de poblaciones rurales, en general se trata de aldeas aisladas con pocos medios de comunicación y deprivación ambiental.

Este estudio revela que los alumnos que ven televisión tienen un rendimiento académico más alto y esto es consistente con numerosos otros estudios.

La educación de la madre es una variable importante que en todos los casos demostró tener significación estadística.

En el caso de Guatemala, una variable que también es importante, es si el alumno o alumna habla o no un idioma maya. En todos los análisis de estos datos esta relación ha demostrado ser de gran importancia. Los alumnos que son bilingües tienen un rendimiento más bajo que los que hablan solamente el español.

Las otras dos variables que resultaron ser buenos predictores del rendimiento, cantidad de libros en el hogar y padres leen periódico, tienen relación con la presencia de diferentes materiales de lectura en el hogar.

\section{Análisis de Correspondencia Múltiple}

Al realizar el análisis del Plano Factorial I-II se evidencia la presencia de dos grupos con características diametralmente opuestas. 
El grupo de bajo rendimiento muestra características predecibles: sobre-edad, asiste a escuelas oficiales que en general están localizadas en el área rural, no tiene educación preescolar y no recibe ayuda en su hogar para hacer tareas escolares debido al bajo nivel educativo de los padres. Otra característica es que no tienen material de lectura en el hogar y no ven televisión. En contraste, el grupo que se encuentra en el otro extremo del plano incluye a alumnos que tienen buen rendimiento escolar, cuya edad corresponde al grado que cursan, asisten a escuelas del sector privado urbano, han tenido oportunidad de asistir a una escuela pre-primaria y reciben apoyo de sus padres al hacer sus tareas. Sus padres tienen mayor nivel educativo y el hogar cuenta con material de lectura, el cual comparten con los niños, y tienen acceso a la televisión.

Si se unen los resultados obtenidos mediante estas dos técnicas estadísticas se obtiene información valiosa, ya que al utilizar los predictores encontrados en los análisis de regresión se puede tratar de modificar algunas de estas variables y obtener así un mejor rendimiento en los alumnos. Por ejemplo, si las madres tuvieran un poco más de información sobre cómo apoyar y motivar a sus hijos en su trabajo escolar, esto redundaría en un beneficio directo para los niños y niñas que habitan en zonas rurales.

Cuando se analizan los factores agrupados en los ejes, al realizar el análisis de correspondencia múltiple, se observa que existe una polarización extrema entre los grupos que fueron evaluados. Esto muestra un sistema social y educativo que presenta una gran desigualdad y que hace que algunos alumnos tengan en su contra todos los factores del hogar asociados al rendimiento, mientras que otros tengan a su favor todos los aspectos asociados, por lo cual, su rendimiento va a ser bueno.

Por otra parte, la mayoría de la población se encuentra dispersa en el medio de los ejes. Es decir, alumnos con un rendimiento regular, cuyos padres tienen alguna educación, pero no le prestan mucha ayuda a sus hijos, tal vez vean televisión algunas veces y asisten a escuelas 
oficiales en sectores urbanos. En general, el rendimiento promedio tanto en lectura como en matemática es bajo, sobre todo de los alumnos de escuelas rurales que representan a la mayoría de la población.

En este trabajo se intentó examinar la relación que existe entre el rendimiento escolar de alumnos de educación primaria y las variables relacionadas con el hogar. La conclusión a la que se llega es que un porcentaje importante de la varianza en el rendimiento, especialmente en lectura, se explica por ciertas variables del hogar. El resto, probablemente, se podría explicar por factores relacionados con el docente y con la escuela en sí o por características individuales de los alumnos.

\section{Referencias}

Baessa, Y., Girón, R., Mejia, M., De Cordón, R., Garcia, L., De Avendaño, L. et al. (1999). Informe de resultados del Proyecto Nacional de Evaluación del Rendimiento Escolar. Guatemala: Ministerio de Educación/Universidad del Valle de Guatemala.

CISIA (1993). Sistema portable para el análisis de datos numéricos. Santiago: Técnicas.

Clarke, A. T. \& Kurtz-Costes, B. (1997). Television viewing, educational quality of the home environment and school readiness. Journal of Educational Research, 90(5), 279-285.

Cooper, H., Valentine, J. C., Nye, B. \& Lidsay, J. J. (1999). Relationships between five after-school activities and academic achievement. Journal of Educational Psychology, 91, 369-378.

Cornejo, J. M. (1988). Técnicas de investigación social: el análisis de correspondencia (teoría y práctica). Barcelona: PPU.

Crivisqui, E. (1993). Análisis factorial de correspondencias, un instrumento de investigación en ciencias sociales. Asunción: Laboratorio de Informática Social, Universidad Católica de Asunción.

Halle T. G., Kurtz-Costes B. \& Mahoney, J. L. (1997). Family influences on school achievement in low-income, African American children. Journal of Educational Psychology, 89(3), 527- 537. 
Laboratorio Latinoamericano de Evaluación de la Calidad de la Educación. (2000). Primer estudio internacional comparativo sobre lenguaje, matemática y factores asociados, para alumnos del tercer y cuarto grado de la educación básica. Santiago: UNESCO. 


\section{ANEXOS}

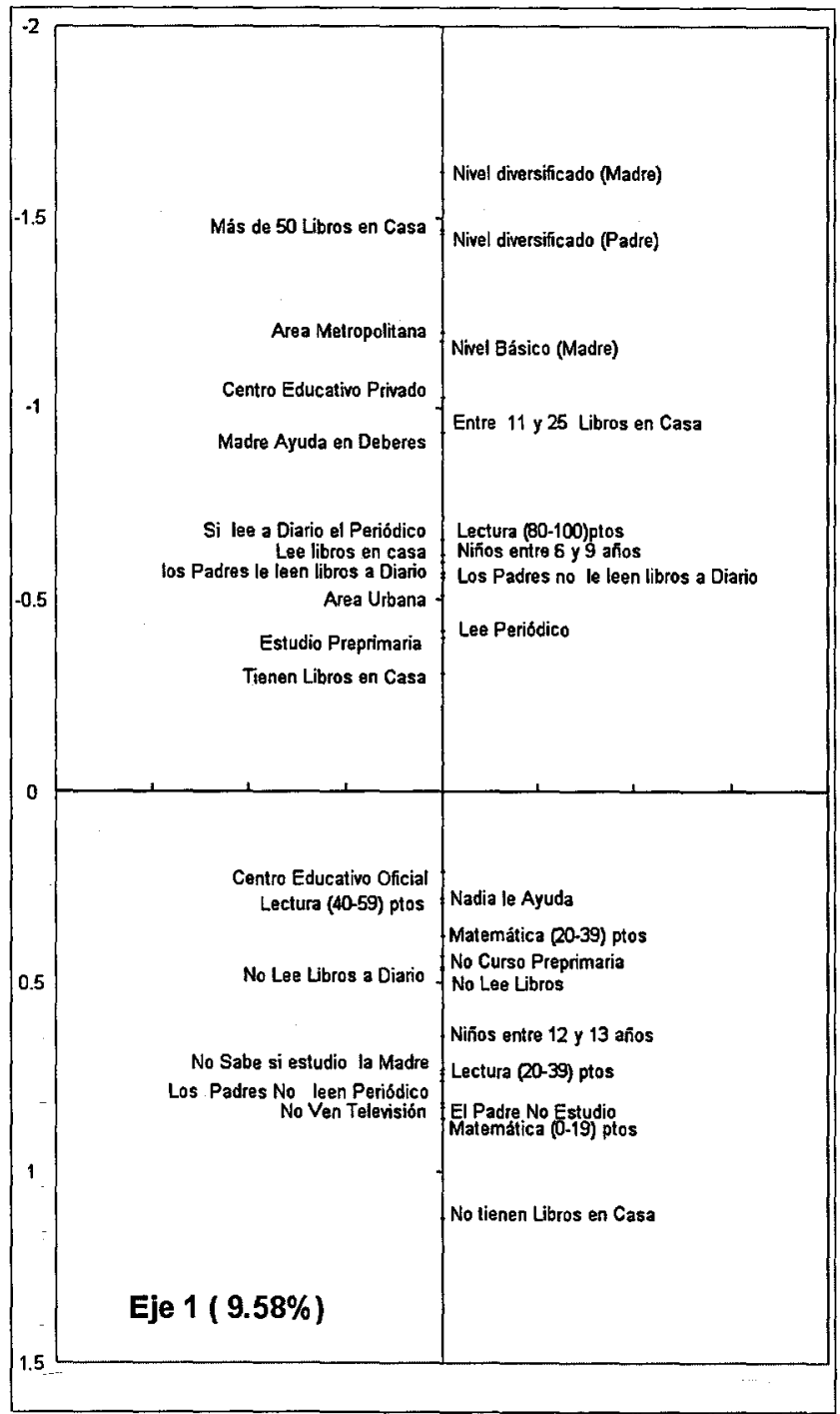

Figura 2. Conformación del eje 1 según contribución absoluta por modalidad. 
Yetilú de Baessa y Francisco Javier Fernández

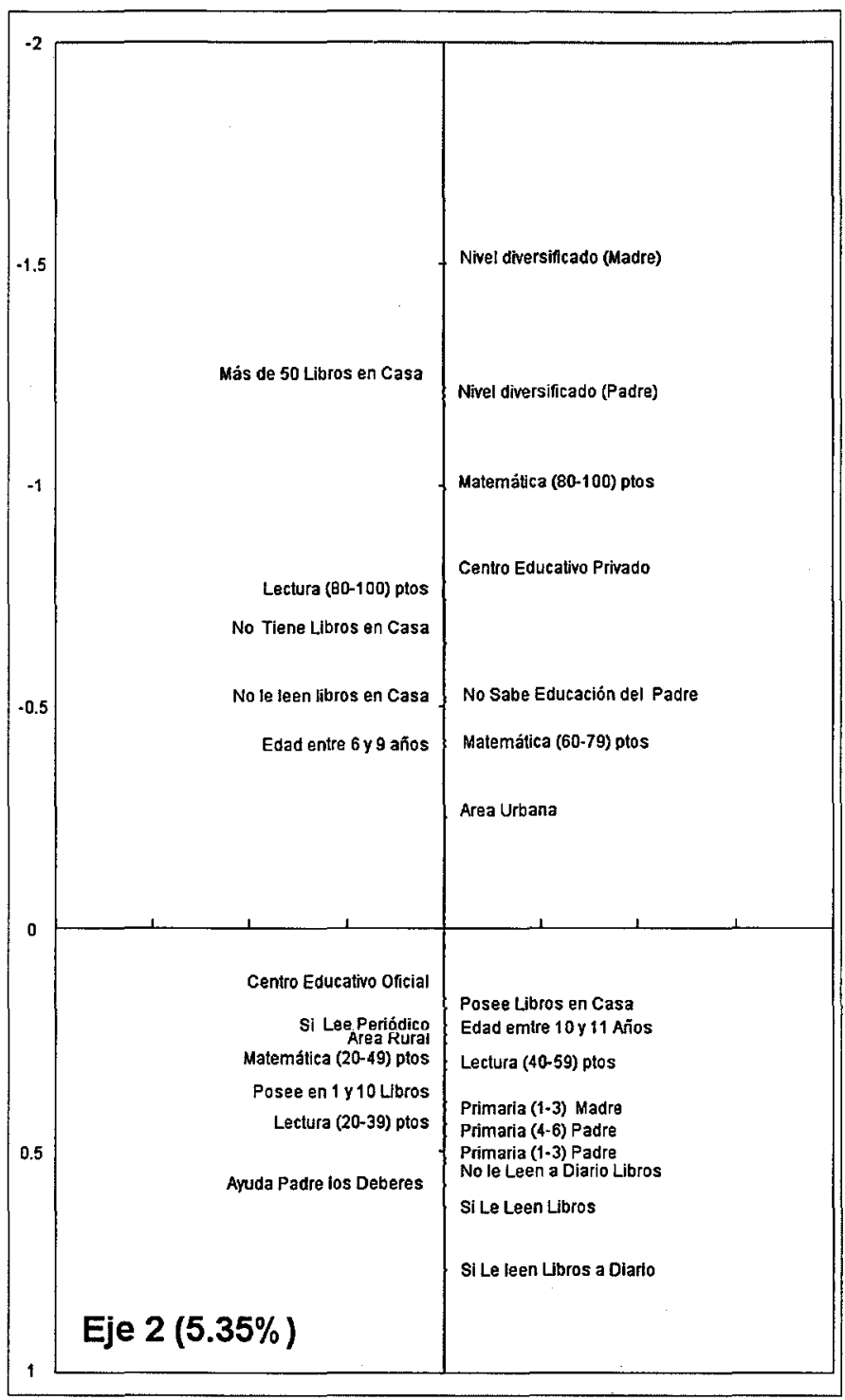

Figura 3. Conformación del eje 2 según contribución absoluta por modalidad. 
Factores del hogar en el rendimiento escolar

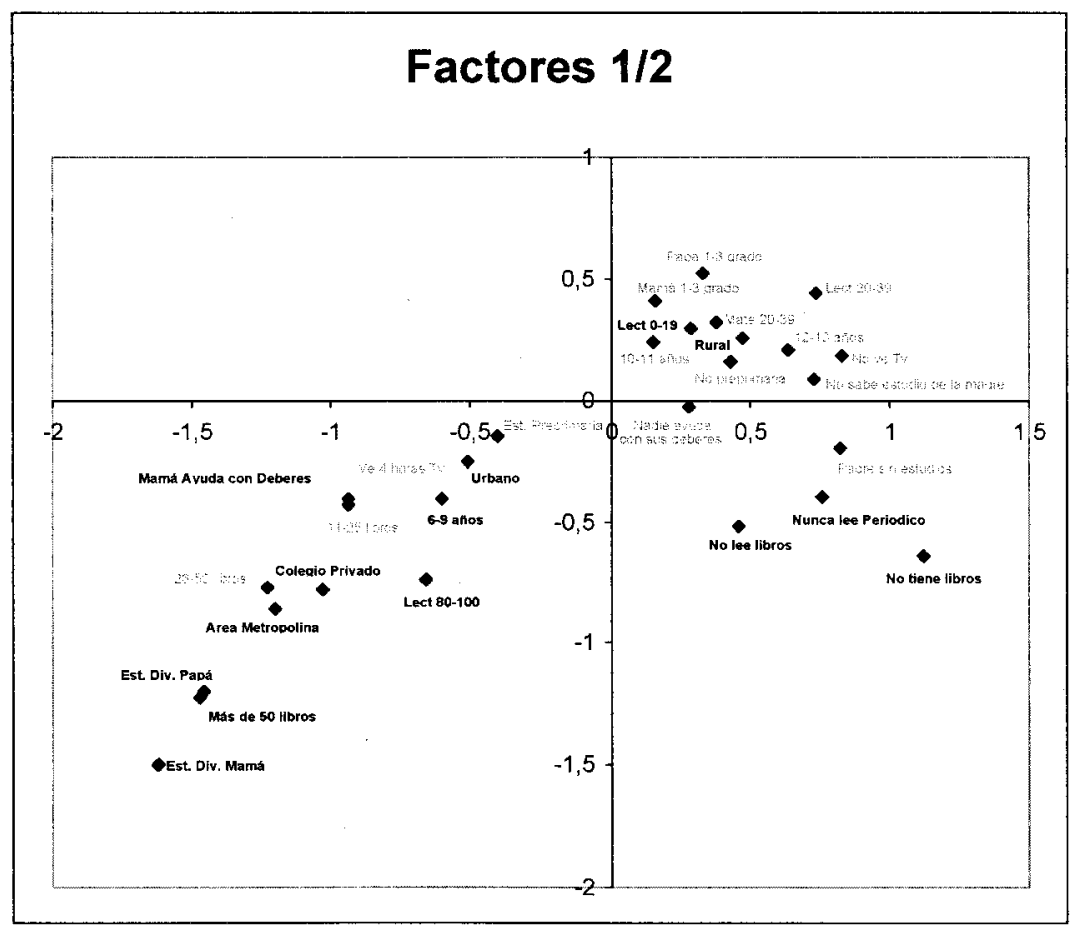

Figura 4. Conformación del plano factorial $1 / 2$ según contribución absoluta por modalidad. 